\title{
Recent developments in ARP/WARP
}

Sravya Mounika Kantamneni ${ }^{1}$, Grzegorz Chojnowski ${ }^{1}$, Joana Pereira ${ }^{1}$, Daria Beshnova ${ }^{1}$, Philipp Heuser ${ }^{1}$, Umut Oezugurel $^{1}$, Victor Lamzin $^{1}$

${ }^{1}$ European Molecular Biology Laboratory (EMBL), Hamburg, Germany

E-mail: skantamneni@embl-hamburg.de

The ARP/wARP software project [1,2] includes an automated model building and refinement for macromolecular crystal structure determination. It is based on the results of two decades of extensive research and development in the areas of Xray crystallography, informatics, data mining, and statistical pattern recognition. With the use of computationally efficient methods ARP/WARP provides easy-to-use pipelines for building models of proteins and their complexes with bound nucleotides and small-molecule ligands.

Here we present recent innovations of ARP/WARP that improve performance at resolutions 3.0 -3.5 $\AA$ and enable interpretation of cryo-electron microscopy (cryo-EM) density maps. The main-chain tracing tools for both proteins and nucleic acids have been advanced to yield more reliable models with improved local stereochemistry. A new protein side-chain docking module has been developed and now provides better performance, particularly at low resolution. This enabled a provision of a new, fully automated tool for building atomic models of proteins and nucleic acids into cryo-EM maps at resolutions better than $3.5 \AA$. Finally, the ARP/WARP web-server has been completely redesigned and provides a more intuitive interface to all the ARP/WARP functionalities. Most recent version of ARP/WARP and the web-service are available at http://www.embl-hamburg.de/ARP/.

[1] Langer, G.G. et. al. (2008), Nat. Protoc. 3(7), 1171-1179.

[2] Langer G.G. et. al. (2013), Acta Cryst. D69, 635-641.

Keywords: Model Building, Refinement, cryo-EM 